\title{
REVISIONES
}

\section{Limitaciones y posibilidades de la pedagogía intercultural para niños indígenas}

\author{
Limitations and possibilities of the intercultural pedagogy for indigenous children \\ Limitações e possibilidades da pedagogia intercultural para crianças indígenas
}

\section{Oscar Lennon del Villar}

Universidad Metropolitana de Ciencias de la Educación, 224124 83, oscar.lennon@umce.cl

\section{RESUMEN}

El tema abordado es la educación intercultural para niños de los pueblos indígenas de América Latina. Se critica el propósito de adecuar los curriculum a la cultura de los niños tomando sólo en cuenta sus conocimientos, o algún otro aspecto de la situación de aprendizaje. En contraposición a ello, se postula que en todos los componentes de ésta se manifiesta la dimensión cultural. Y se examinan, en base a estudios etnográficos, las características más relevantes de los aprendizajes que llevan adelante los niños indígenas en su vida cotidiana. Posteriormente, se analizan las numerosas diferencias que separan estos aprendizajes de los del ámbito escolar, y se analizan además las variaciones culturales de la cognición. Se sostiene que la consideración de todo ello es un requisito indispensable para la implementación de dispositivos curriculares de verdad culturalmente pertinentes.

Palabras clave: educación, indígenas, cultura, aprendizaje, cognición.

\section{ABSTRACT}

This paper deals with intercultural education for children of the indigenous peoples of Latin America. The intention of adapting the syllabi to the culture of the children taking only into consideration their knowledge or some other aspect of their learning situation is strongly criticized. In contrast, it is proposed that in every component of it, cultural dimension is evident. The most relevant characteristics of the learning process that indigenous children develop in their everyday life are analyzed based on ethnographic studies. Subsequently, the numerous differences that separate these learnings from those in school contexts, and the cultural changes regarding cognition are also analyzed. Finally, it is supported that the consideration of all these aspects is an indispensable requisite for the real implementation of curriculums culturally pertinent.

Keywords: education, natives, culture, learning, cognition.

\section{RESUMO}

A questão abordada é a educação intercultural para crianças dos povos indígenas da América Latina. Critica-se o propósito de adequação dos currículos à cultura das crianças, considerando apenas seus conhecimentos ou algum outro aspecto da situação de aprendizagem. Em contrapartida, postula-se que em todos os componentes desta manifeste a dimensão cultural. São examinadas, com base em estudos etnográficos, as características mais relevantes das aprendizagens empregadas pelas crianças indígenas em suas vidas cotidianas. Posteriormente, analisam-se numerosas diferenças que separam tais aprendizagens daquelas do âmbito escolar e as analisam para além das variações culturais da cognição. Sustenta-se que a consideração de tudo isso é um requisito indispensável para a implementação de dispositivos curriculares culturais e realmente pertinentes.

Palavras-chave: educação, indígenas, cultura, aprendizagem, cognição. 


\section{INTRODUCCIÓN}

En las sociedades multiétnicas de América Latina, se han puesto en marcha no pocas experiencias de educación intercultural que tienen por destinatarios a los niños de los pueblos indígenas, cuyas maneras de actuar, pensar y sentir son sensiblemente distintas de las que predominan en la escuela. Por lo cual, cabe decir que esas experiencias representan la forma por excelencia, o más extrema, de la educación intercultural, lo que implica un desafío no menor desde la perspectiva de la pedagogía. Un desafío al que se ha dado respuesta a través de la propuesta de un currículum culturalmente pertinente, es decir, de modalidades de trabajo en el aula que se adecúen a las características culturales de los alumnos de pertenencia indígena. Se trata de un postulado en torno al cual existe un amplio consenso, y que es en realidad difícil de recusar, por una serie de razones: pone a los niños y al aprendizaje en el centro de los procesos del aula, busca generar aprendizajes significativos, toma en cuenta además la identidad cultural de los alumnos. Pero la idea de elaborar formas curriculares pertinentes resulta mucho más discutible en lo que respecta a la manera de darle una traducción práctica. Las más de las veces, ella consiste en la identificación de los conocimientos culturales particulares de los alumnos indígenas, y en el intento posterior de incorporarlos a los procesos de aprendizaje; en otras ocasiones, se toman en consideración las representaciones de la educación de una comunidad indígena y los valores que las orientan, y casos hay también en los que se hace simplemente mención a elementos adicionales, como las formas de comunicación. Pero no hay, al parecer, propuestas en las que se tenga presente la totalidad de los componentes de la situación escolar de aprendizaje y la eventual influencia sobre ellos de la dimensión cultural. A fin de cuenta, esto quiere decir que la pedagogía intercultural no es suficientemente cultural, no ha logrado asumir en su plenitud todas las consecuencias que se desprenden de la existencia de la cultura; esto es, en primer lugar, el hecho de que ella se manifiesta en todos los aspectos y situaciones de la realidad humana. Por lo que no resulta posible restringir su presencia e influencia sólo a ciertos aspectos de una situación de aprendizaje, cualquiera que ella sea.

Es necesario establecer, por consiguiente, que una pedagogía intercultural no puede dejar de situarse en una perspectiva integralmente cultural, y afirmar que las diferencias culturales se actualizan en todos los componentes de cada contexto educacional. Incluyendo en especial uno de estos al que no se ha prestado una atención mayor hasta ahora: las variaciones culturales de la cognición, las que no se limitan ni mucho menos a la esfera de los puros conocimientos, dado "que las personas de diferentes contextos culturales desarrollan diferentes modelos de habilidades y diferentes modos de percibir el mundo y de resolver problemas" (Mishra, 1997: 146). Así lo demuestran los numerosos estudios que ha llevado adelante la sicología cultural en torno al condicionamiento cultural de los procesos cognitivos, de los que no hay casi rastros en las experiencias de educación intercultural. Tal vez porque en el seno de ésta, se sigue asumiendo "de un modo larvado, implícito, que el funcionamiento psicológico básico (...) es previo a cualquier experiencia cultural" (Pozo, 2006: 186), y sólo puede ser modificado por la cultura en aspectos más bien externos y secundarios. Sin embargo, se trata de un punto de vista que ha sido ampliamente refutado por los estudios de orientación cultural, los que han hecho ver que los procesos psicológicos del conocer cobran realidad, operan y se desarrollan en el seno de la vida cotidiana de las distintas culturas, en estrecha relación con las prácticas que allí 
tienen lugar. Y se ha señalado igualmente que la función más esencial de la cognición es la de orientar las acciones e interacciones sociales que las personas están constantemente desplegando cada día, las que a menudo consisten en actividades conjuntas que ponen de manifiesto "la naturaleza compartida de las tareas cognitivas en la vida cotidiana" (Harkness y Super, 1997: 11).

\section{EL APRENDIZAJE EN EL CONTEXTO CULTURAL}

Es también en este marco, la de la acción y de los contextos culturales en que ella siempre se sitúa, que los niños llevan a cabo sus aprendizajes, a través de su participación progresiva en las prácticas sociales de su comunidad, y en las cuales movilizan y desarrollan progresivamente sus saberes y sus capacidades cognitivas diversas. Desde la perspectiva cultural, los niños contribuyen activamente a su propia socialización, ya que las actividades que tienen lugar en su entorno social constituyen su principal centro de interés, tienden a involucrarse en ellas espontáneamente, y a prestar su colaboración cuando tienen la posibilidad de hacerlo. Por cierto, todo esto reviste formas distintas en cada cultura, en relación principalmente con sus prácticas sociales cotidianas y, en especial, con la manera en que está organizada la vida y la actividad de los niños. En este sentido, las comunidades indígenas poseen una característica particularmente relevante que condiciona la experiencia de los niños y sus modalidades de aprendizaje: la posibilidad que tienen de acceder a casi todos los escenarios en que se desenvuelve la vida social de su comunidad, de escuchar y ver casi todo lo que se dice y se hace, de observar casi todas las actividades que allí se realizan. En cambio, en el caso de las formas de vida "modernas" ocurre todo lo contrario, al haber una marcada separación entre el mundo de los adultos y el de los niños, e incluso al hallarse los propios niños separados entre ellos por edades a través de la institución escolar. En esos ambientes sociales, "la segregación de los niños de las actividades de la comunidad adulta forma parte de las evidencias cotidianas" (Rogoff, 2003: 133). Vale decir, por tanto, que la configuración de la existencia y la experiencia del niño es muy diferente en uno y otro caso, tal como lo han podido constatar los trabajos de la sicología cultural en torno al desarrollo, en los que se señala que "no es sólo el contenido de las rutinas culturales lo que varía, sino también el tipo de participación de los niños en ellas" (Harkness y Super, 1997: 9).

Como consecuencia de estas distintas formas de participación en la cultura, las modalidades de aprendizaje de los niños son también muy distintas. En los pueblos indígenas, los procesos de aprendizaje tienen lugar sobre todo en una amplia gama de contextos cotidianos en los que el niño se involucra para observar de cerca y durante todo el tiempo que quiera las actividades de los mayores. En semejantes circunstancias, el soporte principal de la adquisición de saberes y destrezas es el aprendizaje por observación, el que opera en las más distintas esferas de la realidad cultural, como lo ha podido constatar la antropología. Así, en el caso de los nahuas de la Sierra de Puebla en México, que viven dedicados principalmente a la agricultura, nada o casi nada de la actividad de los adultos es ocultado a los niños. Por lo mismo, la observación constituye el método por excelencia de los aprendizajes, y su importancia es subrayada por los propios adultos, los que insisten ante los niños en la necesidad de observar con detención los procesos de los que son espectadores. De acuerdo a Chamoux (1986), observar detenidamente de qué manera se 
hace algo es "el método en el cual más confianza se tiene para lograr una transmisión adecuada de saberes y de los saber hacer. El aprendiz debe mirar cuidadosamente cuáles son los gestos que se hacen, en qué orden, así como sus efectos sobre el material que se está trabajando" (16). De hecho, en los trabajos etnográficos son frecuentes los comentarios en el sentido de que en estos universos sociales pareciera que los niños aprenden casi todo solos, mediante su implicación precoz en los diversos escenarios sociales de su cultura. Desde su más tierna edad su madre lo lleva consigo en sus actividades de cada día, en una posición que le permite observar lo que acontece en su entorno, y le brinda a la vez la ocasión de interactuar con quienes se acercan a ella. Y su interés por todo lo que ocurre en su universo social se acrecienta cada vez más, como lo muestra el ejemplo de los niños xavante de la región amazónica del Brasil. Apenas están en condiciones de alejarse de su casa se unen a otros niños que recorren en grupo la aldea observando lo que allí sucede, de manera que "hay muy pocas cosas que escapan a su atención, y su curiosidad es insaciable. Desde que ven que alguien va a algún lugar lo llaman para preguntarle adónde va" (Maybury-Lewis, 1992: 122); y la respuesta que le dan los mayores es casi siempre muy precisa, a fin de impedir que los niños puedan seguirlos.

Las características particulares que asume la existencia cotidiana para los niños indígenas se manifiesta, asimismo, en sus restantes formas de aprendizaje, las que están también conectadas con sus posibilidades de observar casi todo lo que acontece en su comunidad. Tal es el caso de los juegos de imitación del quehacer de los adultos, como lo muestra el ejemplo de los niños jivi, un pueblo que ocupa una amplia región de sabanas y bosques entre Colombia y Venezuela. A una temprana edad, antes de los cuatro años, "el niño observa al padre construir su arco y lo imita, hace su arco" (Amodio, 2005: 256) con el que juega a emular las prácticas de caza de su padre. Las niñas de la etnia warao, que habita en el delta del Orinoco, en un lugar de una exuberante vegetación, "juegan desde una temprana edad a curar a sus hermanitos con hierbas que han visto utilizar a su madre" (Amodio, 2005: 442). Aprenden así, primero a distinguir las distintas especies de plantas, y a identificar posteriormente, con el apoyo de su madre o de su abuela, cuáles son las que necesitan utilizar en cada ocasión. A veces, cuando necesario, llegan a convertirse en fitoterapeutas expertas, capaces de movilizar toda una serie de saberes complejos sobre las funciones curativas específicas de cada planta, las combinaciones que es conveniente hacer, o incluso sobre sus olores, ya que hay plantas a las que se les atribuyen propiedades sanadoras.

Sin embargo, no es sólo mediante la observación de las actividades de los adultos que los aprendizajes de los niños indígenas toman forma, sino también mediante la participación en ellas, en un tipo de contextos en los que tienen la oportunidad de emprender tareas conjuntas con las personas de un nivel más avanzado. Los niños varones del pueblo añu, que vive en una plataforma palafítica situada sobre una laguna, al norte del estado de Zulia en Venezuela, desde los cinco o seis años empiezan a acompañar a su padre en trabajos de pesca de cangrejos y de peces. Consiguen así alcanzar el dominio de las técnicas de pesca y adquieren numerosos conocimientos que guardan directa relación con estas prácticas y con su forma de vida lacustre: en especial, sobre las zonas de mayor abundancia de peces, las corrientes que circulan en la laguna, los cambios que se producen en ellas y sus consecuencias diversas, (Amodio, 2005). No obstante, entre los niños añu las actividades de captura de peces y de cangrejos también se efectúan de otra manera, en otra clase de contextos sociales, en grupos compuestos por niños de diferentes edades, en que los más 
grandes asustan a los peces golpeando en el agua, en tanto que los más pequeños los atrapan en redes que mantienen sumergidas.

Participar en actividades conjuntas con sus pares, en las situaciones socialmente estructuradas de su cultura, es otra de las modalidades características de aprendizaje de los niños indígenas, los que en ciertas culturas suelen pasar gran parte de su tiempo entre ellos. En una comunidad maya de Guatemala, estudiada por Rogoff, "quedan fuera del control directo de los adultos a los 3 o 4 años, entonces se mueven por el pueblo en grupos que incluyen niños de distintas edades, se divierten solos observando lo que ocurre e imitando a los mayores en sus juegos" (Rogoff, 1993: 163). Además, hay también juegos o formas de entretención específicamente infantiles, que se prosiguen a veces en la adolescencia, como los duelos de improvisación verbal a los que se libran en pareja niños y jóvenes chamulas, una comunidad maya de México. En cada turno de palabra uno de los dos contendientes retoma la frase pronunciada anteriormente por su oponente, introduciendo en ellas ligeras modificaciones para proporcionarle así una réplica, en una serie de ofensas rituales recíprocas que se prolongan a veces durante varios centenares de intercambios. Desde los dos o tres años los niños empiezan a ser partícipes de estos desafíos, lo que les permite desarrollar "elegancia y poder en el uso del lenguaje" (Rogoff, 2003: 252), destrezas que se manifiestan en plenitud en el tiempo de la adolescencia y que son altamente valorizadas en su cultura. De manera similar, los niños y jóvenes aymaras se enfrentan en un juego que requiere el uso de estrategias cognitivas complejas, "el zorro y las ovejas", el que se practica dibujando en el suelo un diagrama compuesto por un cuadrado al que se añade un triángulo en su borde superior externo; la primera de estas figuras representa la pradera de las ovejas, la segunda la gruta del zorro, cuyo objetivo es comerse a las ovejas, las que a su vez intentan inmovilizarlo en su gruta o desalojarlo de ella; en cada movimiento, los jugadores desplazan sus peones a través de una serie de puntos que se sitúan al interior de cada una de las dos figuras geométricas (Romero y Gottret, 1999).

A propósito de todas estas experiencias de aprendizaje cultural, que se apoyan en la observación por los niños de las prácticas de los mayores y en su participación en ellas, en los juegos de imitación y las tareas que realizan junto a sus pares, importa recordar dos conclusiones centrales de la sicología cultural. Primeramente, el hecho de que en ellas los niños adquieren no sólo saberes de naturaleza procedimental para abordar problemas específicos, sino también conocimientos de carácter conceptual, "un rico cuerpo de conocimientos sobre tópicos de carácter científico" (Schliemann et al. 1997:189). En segundo término, que es en el marco de esta clase de situaciones socialmente organizadas que tiene lugar el desarrollo intelectual de los niños, a través de su progresivo involucramiento en ellas, en actividades que realizan junto a personas de un nivel más avanzado. De ahí que se haya sostenido que, a fin de cuentas, el desarrollo cognitivo "es un aspecto de la transformación de la participación de las personas en actividades socioculturales" (Rogoff, 2003: 237). Es decir, consiste básicamente en una ampliación progresiva de las posibilidades de acción y de comprensión de los niños, a medida que se van integrando a nuevos contextos y prácticas de su mundo social, o que prosiguen su participación en ellos con un grado de pericia cada vez mayor.

Un ejemplo particularmente demostrativo de esto es el caso de los niños mazahua, que acompañan a sus madres y otros adultos a un mercado de México donde se instalan con puestos de venta de verduras. Durante varias semanas, una niña pequeña pasa su tiempo junto a su madre, "emulando todos los movimientos de preparación, de cuidar y de vender" 
(Paradise, en línea, 2005: 14); de este modo, va a construyendo gradualmente las destrezas que le permiten asumir tareas de este tipo cada vez más numerosas: sacar las capas externas de las cebollas, ponerlas ordenadamente sobre una superficie, e incluso instalar su propio puesto utilizando unos cartones que se hallan abandonados. Esto constituye una ilustración del hecho de que los niños buscan involucrarse de manera activa y espontánea en las actividades sociales de su comunidad, e intentan ayudar en las tareas que los adultos realizan. Es lo que deja entrever, igualmente, en el mismo escenario del mercado, una niña de alrededor de ocho años, que es capaz a esa edad de quedarse sola atendiendo un puesto de verduras. Una tarea que asume con un sentimiento profundo de alegría y entusiasmo, que es expresión de la motivación intrínseca propia de esta clase de aprendizajes que tienen lugar en situaciones reales de la vida social, que están antecedidos por la oportunidad de observar a diario y de cerca lo que allí se hace, que procuran al niño la posibilidad de colaborar en tareas que son de vital importancia para su comunidad, y que le resultan por lo mismo altamente significativas.

Por cierto, los contextos de aprendizaje de los niños de los pueblos indígenas no son solamente los que se han mencionado, ni se circunscriben a los de orden productivo, ya que el aprendizaje está presente en todas las esferas y niveles de la vida social. Así, entre los warao, los niños aprenden los cantos con que los mayores acostumbran acompañar sus faenas cotidianas, y a tocar instrumentos como flautas, maracas y tambores que se usan en ceremonias religiosas, en rituales y en celebraciones festivas (Amodio, 2005). A lo que se suman actividades de intención decorativa o estética, como los dibujos con que se adornan cestas, vasijas u otros objetos, además de las pinturas y los adornos corporales, a los que en ciertas culturas se concede una especial atención, como por ejemplo entre los bororo del Mato Grosso, en donde "el gusto por los ornamentos es tan acentuado que los hombres están continuamente componiendo aderezos" (Lévi-Strauss, 1950: 263) para ellos. Y están también los aprendizajes de orden social y simbólico, en relación a los cuales juegan un papel importante las narraciones que hacen los adultos. En el pueblo mapuche, los relatos orales son un instrumento cultural importante para adquirir el conocimiento de las relaciones de parentesco, de los preceptos morales y de los valores en que se sustentan, lo que es también enseñado en el contexto de actividades socioreligiosas, como el We xipantu, el año nuevo mapuche (Quintriqueo, 2005). También constituyen un momento privilegiado del aprendizaje de la cosmovisión cultural, que se transmite por intermedio de distintos tipos de narraciones: los epew, que ponen en escena casi siempre a animales, y que además de hacer reír a los oyentes, constituyen un medio de transmisión de valores, como el respeto por la naturaleza; los ngütxam, en los que se describen hechos de un tiempo cercano o remoto, considerados reales; y, finalmente, los perrimontún, que "hacen referencia a fenómenos sobrenaturales que afectan a la comunidad o las personas" (Quilaqueo, 2005: 78).

Como se desprende de lo anteriormente expuesto, no son pocas las características particulares que asumen los aprendizajes de los niños en el mundo indígena, en conexión con un modo de vida infantil que les proporciona la posibilidad de observar y participar en las distintas situaciones en que se desarrolla la vida social de su comunidad. Por cierto, es un hecho de primera importancia para una pedagogía intercultural pertinente, sobre todo si se considera que el aprendizaje es el eje estructurante de toda situación educativa y es, al mismo tiempo, la finalidad hacia la cual ésta apunta. Todo ello cobra aún mayor relevancia si se considera, además, que esas modalidades de aprendizaje son muy distintas 
de las habituales en el ámbito escolar, puesto que no se circunscriben a momentos y lugares precisos ni siguen un curso predeterminado, sino que se inscriben en el curso cotidiano de la vida social y en sus más diversos contextos. Es, pues, un desafío no menor para la pedagogía intercultural el de crear situaciones de aprendizaje que sean coherentes con las formas específicas de aprender de los niños de las culturas indígenas. Sin embargo, esto implica entrar a considerar de manera más precisa las características de esos aprendizajes, pues son fenómenos complejos que cubren una variedad de aspectos diferentes en los que también se manifiesta la dimensión cultural, de los que no es posible por lo tanto prescindir.

\section{APRENDIZAJES COTIDIANOS Y APRENDIZAJE ESCOLAR}

Si desde la perspectiva de la elaboración de dispositivos curriculares pertinentes, resulta necesario identificar las múltiples facetas culturales particulares implícitas en los aprendizajes de los niños de los pueblos indígenas, es indispensable también analizar las diferencias que las separan de las modalidades específicas de aprendizaje que son características del ámbito escolar. Desde luego, las diferencias son numerosas. En primer lugar, el hecho de que el niño indígena desempeña un papel central o protagónico en los aprendizajes, los que suele abordar las más de las veces por su propia iniciativa, como consecuencia de la posibilidad que tiene de estar presente, observar y participar en casi todos los escenarios de su universo cultural. Por lo mismo, la intervención de los adultos suele ser discreta e intermitente, poco visible, e incluso los padres suelen "esperar que los niños aprendan por su propia cuenta, con una instrucción específica reducida, pero en un trasfondo general de expectativas sociales que logren ajustarlos a las normas de la comunidad" (Super y Harkness, 1997: 18). Al revés de lo que ocurre, según los mismos autores, en otros ámbitos sociales como las ciudades, en donde hay prácticas explícitas para aprender y enseñar, los niños se hallan segregados casi por completo de la vida diaria de los adultos, lo que obliga a estos a comprometerse mucho más en los aprendizajes de sus hijos, convirtiéndose incluso en sus compañeros de juego y entretención. Lo que demuestra, por añadidura, que la responsabilidad que asumen el niño y el adulto en los procesos del aprendizaje infantil no es la misma en las distintas culturas, como resultado principalmente de la forma en que está configurada la experiencia infantil en cada una de ellas.

Por otro lado, en el universo indígena, el aprendizaje de los niños tiene lugar casi siempre en el contexto mismo de la acción, participando en actividades socialmente relevantes que son de un particular interés para ellos y les permiten realizar una contribución importante a su comunidad, lo que les vale el reconocimiento de quienes la integran. Se trata por lo mismo de prácticas impregnadas de significaciones múltiples y constantemente presentes, lo que también se manifiesta en los procesos cotidianos de aprendizaje. Como lo señala la sicología cultural, "la significación es el aspecto más importante y destacado en la resolución de problemas de cada día" (Schliemann et al. 1997: 202), y es en función de ella que el niño está continuamente operando cuando hace frente a esta clase de tareas, lo que difiere considerablemente de lo que casi siempre ocurre en la escuela. Lo mismo cabe decir a propósito del aprendizaje mediante ensayo y error, el que es escasamente utilizado en los pueblos autóctonos, porque en las situaciones reales los errores casi siempre tienen un costo que puede ser muy elevado, lo que explica también por qué son de común ocurrencia 
en el ámbito escolar. En cambio, en el mundo indígena sí es frecuente el aprendizaje por andamiaje, en el cual una persona experta en una tarea guía y presta apoyo gradual al aprendiz, reduciendo paulatinamente su ayuda a medida que se incrementa el nivel de dominio de éste. Es, pues, una forma de aprendizaje que reduce de manera apreciable la posibilidad de cometer errores, y que favorece al mismo tiempo la continuidad de tradiciones que se intenta colectivamente mantener.

Es esto justamente lo que hace ver una investigación realizada por Greenfield y Childs (2005), en torno al aprendizaje del tejido de las niñas mayas zinacantec, el que reviste un especial interés por el hecho de que se extiende a lo largo de un tiempo prolongado y se sustenta en distintas formas de aprendizaje que se encadenan secuencialmente. En efecto, ya muy pequeñas las niñas dedican una buena parte de su tiempo a observar a las personas expertas, que son siempre mujeres, mientras están realizando el trabajo de tejer en un telar de cintura, heredado de la antigua civilización maya. Más adelante, se dedican a tejer en un telar de juguete muy semejante al telar real, pero que es más pequeño y más fácil de entender y de utilizar. Así, pueden continuar desarrollando sus habilidades en una edad en la que no están todavía en condiciones de manejar adecuadamente los diversos componentes del telar, ni han alcanzado tampoco el dominio de las operaciones que esto supone, las que sí están en condiciones de practicar por separado en el telar de juguete. Hacia los nueve años, las niñas empiezan a tejer de verdad en el telar de los adultos, bajo la guía de una maestra con la que se tiene una relación íntima y personal, pues se trata de su madre, una hermana, una tía o una prima. En la instrucción que éstas proporcionan, el nivel de dificultad se va graduando paulatinamente, y se le presta ayuda a la niña cada vez que la necesita, porque ahora se trata de elaborar productos para ser usados en la comunidad, por lo cual "la importancia del éxito en la terminación (...) aumenta el coste de los errores y hace que el experto esté más pendiente del alumno" (Greenfield y Childs, 2005: 9). Como consecuencia de ello, y de la autoridad de que goza la maestra por ser una persona afectivamente cercana, la niña es "guiada para tejer exactamente de la misma manera que sus predecesoras" (Op. Cit.: 11). Es decir, no existe casi la oportunidad de hacer una práctica mediante ensayo y error, la que según las autoras tiende a favorecer la innovación, que en este caso se contrapone a los valores colectivos que orientan hacia la conservación de la tradición.

No está de más agregar que, en el estudio recién señalado, se hace un análisis también de las diferencias que se dan entre el aprendizaje del tejido de las niñas mayas zinacantec y el que es característico del universo escolar. Primeramente, el hecho de que las niñas tienen la oportunidad de observar durante largo tiempo las tareas que les corresponderá aprender más adelante, y de empezar a practicarlas a través del juego. Además, tienen con su maestra una interacción cercana, de persona a persona, y es la aprendiza la que ocupa constantemente el lugar central en esa relación, en tanto que en la escuela hay numerosos alumnos para un maestro, y si "los alumnos deben mirar y atender al maestro, al tejer la situación es la contraria, siendo la maestra quien debe mirar y atender a la aprendiza" (Greenfield y Childs, 2005: 8). Y también los resultados que se buscan son distintos, ya que cuando las niñas empiezan a tejer en el telar, hacia los nueve años, es con el fin de producir productos reales y útiles, al contrario de lo que pasa en la escuela.

De igual manera, un aspecto adicional de los aprendizajes que llevan adelante los niños indígenas, de particular importancia para una pedagogía intercultural, dice relación con el papel del lenguaje. En este sentido, se ha podido constatar que su uso suele ser bastante 
reducido, comparativamente a lo que ocurre en los universos sociales "modernos" y en la propia institución escolar. Igualmente, se ha señalado que las variaciones culturales también se manifiestan en "la significación social de los distintos tipos de intercambios verbales, y de su grado de elaboración o sofisticación” (Bril y Lehalle, 1988: 59). Así, hay pueblos que tienen un acentuado aprecio por la elocuencia, por el uso de un estilo elaborado de lenguaje, como sucede entre los chamulas, antes mencionados, en donde los niños se ejercitan desde pequeños en la adquisición de esta clase de aptitudes a través de los desafíos verbales en que participan con sus pares. Sin embargo, se ha comprobado que no existe una relación entre las actitudes culturales hacia el habla y el uso de ésta por lo adultos en el contexto de los aprendizajes infantiles. Es lo que muestra el ejemplo de los nahuas de Sierra de Puebla, en México, los que han "conservado desde los tiempos precolombinos el gusto por el buen lenguaje, especialmente en las relaciones de cortesía, en las ceremonias y en los cultos (...). Entablar una charla con un visitante es una obligación" (Chamoux, 1986: 227). No obstante, en las situaciones de aprendizaje, el uso de explicaciones verbales es muy reducido, por lo que cabe concluir que se trata no de una actitud hacia el lenguaje propiamente tal, sino de un rasgo específico de esta clase de situaciones sociales. De hecho, el aprendiz no formula preguntas, y el sujeto que ejerce el rol de instructor no proporciona explicaciones, no emite tampoco comentarios sobre las tareas que se están realizando, ni aporta indicaciones sobre recetas o algoritmos que se podrían utilizar. Se trata, por lo demás, de una modalidad cultural de proceder que se exterioriza no sólo en la adquisición de las técnicas agrícolas, artesanales o culinarias, "sino también en la transmisión de métodos chamánicos que consisten ante todo en un saber de orden verbal" (Chamoux, 1986: 227).

La situación es sólo ligeramente distinta en otros grupos nahuas. Entre los de Cuacuila, por ejemplo, el uso del lenguaje en relación a los aprendizajes está un poco más presente, aunque de una manera elíptica y pausada. Es lo que se puede ver con respecto al arte de bordar que se transmite antes de la adolescencia desde la madre a la hija, o de la hermana mayor a la menor (Chamoux, 1978). En un primer momento, hacia los once años, la niña empieza a realizar tareas relativamente simples, como realizar un bordado sobre una servilleta, empleando todo el tiempo que sea necesario. Posteriormente, la maestra le hace una demostración para que ella realice por sí sola la tarea de bordar blusas grandes y verdaderas. En esta circunstancia, la maestra permanece cerca de ella, pero sólo mira de tarde en tarde lo que está haciendo, y cuando critica o aprueba lo hace mediante un simple gesto, una sonrisa, una palabra o una broma, pero nunca recurriendo a frases largas, y sin que haya tampoco una exigencia de tiempo para finalizar el trabajo.

Sin embargo, las prácticas de comunicación que tienen lugar en los procesos de aprendizaje cubren también otros aspectos que no se han mencionado. Como es el caso de los canales de comunicación utilizados, del uso de preguntas y explicaciones, o de quiénes son las personas con las que los niños habitualmente interactúan, las que en algunas comunidades son sobre todo los hermanos y/o el grupo de pares. Así, las formas de comunicación no verbal -como gestos, miradas, sonrisas, expresiones faciales y contactos corporales- son recurrentes en los intercambios sociales que tienen lugar en pueblos indígenas, especialmente en la relación de la madre con los niños, como consecuencia de la proximidad física y social que caracteriza la convivencia cotidiana de las personas. En cuanto a las preguntas que los niños dirigen a los adultos, no suelen ser frecuentes en el universo indígena, ya que existe la posibilidad de adquirir conocimientos y destrezas mediante la observación directa y prolongada; aunque también hay culturas en las cuales la 
pregunta que un niño formula a un adulto es vista como un intercambio agresivo, que desde pequeño el niño aprende a evitar. Asimismo, en las sociedades indígenas "las interacciones sociales del niño con sus cuidadores se insertan en un contexto en que los intercambios sociales poliádicos son la norma" (Mohanty y Perregaux, 1997: 226); esto, incluso en las interacciones con la madre, contrariamente a los intercambios de naturaleza diádica que mantienen en las culturas "modernas". Además, a menudo se considera que alabar a los niños es una práctica que puede perturbar su relación con su comunidad, que les provoca por tanto un perjuicio, al igual que las conductas en que el niño trata de sobresalir o de entablar relaciones de competencia con sus pares, o intenta exhibir un aprendizaje antes de que esté consolidado. Desde luego, todos esos aspectos de las prácticas de comunicación y relación social que acompañan los procesos de aprendizaje en los pueblos indígenas se hallan bastante alejados de los que son propios de la escuela, situándose en el polo opuesto de éstos.

Como se puede apreciar, son numerosas las diferencias que existen entre las experiencias de aprendizaje de los niños de comunidades indígenas y las que son típicas del mundo escolar. Sin embargo, es un aspecto de la educación intercultural al que no se ha prestado suficiente atención, probablemente por el hecho de que en el seno de ésta no se ha logrado efectuar un descentramiento efectivo respecto a las modalidades escolares del aprendizaje. En otras palabras, se suele pasar por alto el carácter culturalmente situado de los dispositivos institucionalizados de educación, que siempre son el producto de pautas culturales particulares, que no son por tanto culturalmente neutras, y que se manifiestan en los más distintos aspectos de ellos: el tipo de saberes que son objeto de enseñanza, las prácticas de aprendizaje que se utilizan, la forma de participación de los alumnos, las modalidades de interacción y comunicación social, entre otros.

\section{VARIACIONES CULTURALES DE LA COGNICIÓN}

Uno de los elementos que mayor importancia tiene para la puesta en marcha de prácticas curriculares pertinentes radica en las modalidades culturalmente diferenciadas de la cognición humana. Algo que ha sido reiteradamente comprobado por la sicología cultural, la cual hecho ver que los mecanismos de base de la cognición son universales, pero poseen la propiedad de existir y manifestarse bajo una forma particular en cada cultura (Cole, 1999). Además, esas variaciones se manifiestan en los distintos componentes funcionales de la cognición, como la atención, la percepción y la memoria, en el razonamiento y la categorización. En lo que atañe a la atención, los niños mayas exhiben desde pequeños la capacidad de prestar atención y monitorear varias eventos al mismo tiempo, lo que es de particular relevancia cuando el vector más importante del aprendizaje radica en la observación cotidiana de situaciones sociales, en las cuales, dado su carácter poliádico, es frecuente que ocurran varios acontecimientos al mismo tiempo, de suerte que un "estrecho foco de atención hace mucho más difícil darse cuenta de acontecimientos que pueden resultar de un gran interés" (Rogoff, 2003: 321). Además, sus propias madres los acostumbran desde una edad temprana a fijarse atentamente en las actividades que ellas desarrollan, es decir, a poner en práctica este tipo de procesos atencionales, que difieren de manera sensible de los que predominan en el medio cultural "moderno", consistentes en un uso alternado de la atención hacia los distintos eventos sociales en curso. 
De manera similar, "la experiencia de la cultura está íntimamente imbricada en la textura de nuestras percepciones" (Boesch, 1995: 43), pues siempre están mediatizadas por las categorías y significaciones de cada cultura, que guardan relación a su vez con sus prácticas más importantes. Así, por ejemplo, allí donde la agricultura ocupa un lugar central, la capacidad de distinción de las informaciones visuales es de especial relevancia, y los niños dan muestras de habilidades particulares en este dominio que se acrecientan progresivamente con la edad (Maccoby y Modiano, 1980). En lo concerniente a la percepción auditiva, los tapirapé, que habitan en medio de la espesa jungla tropical del centro del Brasil, lo que impide la visión en perspectiva, se guían durante sus partidas de caza en base a los sonidos que salen de la jungla, lo que "les permiten conocer de manera bastante precisa la localización de los animales" (Bossel-Lagos, 1989: 117). Más en general, se ha hecho ver que "todas las culturas disponen de ciertas heurísticas para organizar la información espacial y recordar la ubicación de los lugares" (Mishra, 1997: 164), algo que confirma el mismo ejemplo de los tapirapé, que en sus largos recorridos por la selva consiguen ubicarse y llegar sin dificultad a su destino; y esto, gracias a un sistema cultural de orientación espacial compuesto de múltiples elementos interrelacionados: la posición del sol, los nidos de ciertas aves, las colmenas de abejas salvajes, las madrigueras de los animales, entre otros.

En lo que respecta a los sistemas de clasificación, en las sociedades indígenas estos suelen estar estrechamente asociados a sus numerosos y elaborados conocimientos sobre el entorno ecológico en que transcurre su existencia, como la antropología lo puso en evidencia. Los aymaras del altiplano de Bolivia, especialistas del cultivo de la papa, de especial importancia a una altitud elevada donde el maíz no alcanza a madurar, distinguían y nombraban más de 250 variedades, las que todavía perduran en su vocabulario. Cada una de las cuales se divide a su vez en sub-variedades de acuerdo a distintos criterios, como la forma, la textura o el hecho de que sean "inmediatamente comestibles después de la cocción, o solamente después de una serie de congelamientos y de fermentaciones alternadas" (Lévi-Strauss, 1990: 72). Por su parte, la sicología cultural ha efectuado no pocos estudios sobre las modalidades y criterios de clasificación usados cotidianamente por las personas de distintas culturas La principal conclusión que emerge de ellos se refiere a la influencia de la experiencia escolar, que inclina de manera sistemática a agrupar los objetos, apoyándose en propiedades de carácter semántico, es decir, en el lenguaje; en cambio, los sujetos sin o escasa instrucción escolar tienden a categorizar en base a criterios prácticos y funcionales (Cubero, De La Mata, 2005). Esto no impide, sin embargo, que puedan efectuar clasificaciones de orden más general, cuando resulta necesario. Lo que ha sido comprobado por las propias investigaciones en torno a la categorización, las que han llevado a "refutar la idea de que ciertos grupos culturales muestran menos abstracción o generalidad en los procesos cognitivos comparativamente a otros" (Mishra, 1997: 159).

En todos los demás componentes de la cognición, se ha podido comprobar igualmente su indisociable conexión con la cultura, y las prácticas particulares que la constituyen. Así, a propósito de la memoria, se ha hecho ver que "cuando las estructuras de la actividad son similares (...) las diferencias culturales en los procesos de recuerdo parecen ser mínimas" (Cole, 1999: 74). Por otro lado, las modalidades de rememoración no son las mismas en todas las culturas, hay algunas en las que se usan estrategias de rememoración visual, en tanto que en otras se recurre sobre todo a las de carácter verbal. Así, los niños mayas de una comunidad de Guatemala exhiben una habilidad particular para recordar información 
espacial contextualmente organizada, pero no ocurre lo mismo con unidades aisladas de información presentadas en una lista, las que son recordadas con relativa facilidad por los sujetos que cuentan con experiencia escolar, más aún cuando el uso de la memorización está más presente en sus escuelas (Rogoff, 2003). También hay culturas en las que los elementos a recordar suelen estar entretejidos en el contexto de narraciones, a propósito de lo cual se ha hecho ver que las estructuras o "gramáticas de historias difieren en su forma a través de las culturas y estas diferencias conducen a distorsiones en el recuerdo de historias que no encajan con el ideal" (Rogoff, 1993: 89) narrativo de cada cultura.

De igual modo, se ha observado que la manera de abordar y resolver los problemas no es la misma en las distintas culturas, ya que cada una de éstas le imprime una orientación particular, de acuerdo a lo que son sus valores más centrales. Hay culturas en las que es "valorada una aproximación holística más que analítica en la resolución de problemas, y la deliberación antes que la prisa es considerada como un curso de acción apropiado. Y (...) la discusión colectiva en vez de la reflexión individual puede ser preferida" (Mishra, 1997: 148). En cambio, en otros universos culturales son muy distintos los criterios y valores en función de los cuales se define cuál es la forma más apropiada de dar solución a los problemas, e incluso de lo que puede ser considerado como un problema. En el caso de la escuela, se trata de problemas de un tipo muy particular, de carácter puramente intelectual y autosuficientes, sin relación con las actividades y situaciones de la vida de las personas, y que es necesario resolver con rapidez, en forma individual y con el máximo de precisión posible. Todo lo cual pone de relieve, por lo demás, el hecho de que la cognición se despliega y toma forma en actividades socialmente estructuradas de la cultura, de las que son una parte constitutiva las modalidades particulares de relación e interacción social que en ella prevalecen.

Por consiguiente, la pedagogía intercultural no puede dejar de lado el hecho de que la cognición adopta modalidades particulares en cada cultura, las que se manifiestan en todos sus componentes funcionales. De esto, se desprende que las maneras de pensar de los niños indígenas pueden no ser coincidentes con las que se usan y son validadas en la escuela, lo que representa un obstáculo mayor para sus procesos de aprendizaje. Sin embargo, es algo que no ha sido tomado en cuenta hasta ahora, a pesar de que tiene una indudable relevancia, ya que los niños entran en relación con las tareas y problemas del ámbito escolar por medio de los saberes y las destrezas cognitivas que han adquirido en su propio universo cultural. Por lo anterior, para la pedagogía intercultural resulta indispensable lograr apartarse de las concepciones uniformistas de la cognición que han predominado por largo tiempo, tanto en la escuela como fuera de ella. Es indispensable que ella esté en condiciones de entender que los niños de culturas diferentes tienen maneras de pensar distintas, apoyándose para esto en los saberes generados desde la antropología y la sicología cultural, las que deben convertirse en el referente disciplinario permanente de su quehacer.

\section{CONCLUSIONES}

Cabe decir, en síntesis, que una pedagogía intercultural para los niños de las comunidades indígenas no puede dejar de situarse en una perspectiva integralmente cultural, lo que implica ante todo tener presente tres cosas: primero, las características particulares que asumen los aprendizajes en su universo de pertenencia; segundo, la naturaleza cultural de 
las modalidades escolares de aprendizaje, así como las múltiples diferencias que separan a éstas de los aprendizajes que llevan a cabo los niños indígenas; tercero, las variaciones culturales de la cognición.

Sólo de esta manera, basándose en la consideración efectiva y conjunta de estos tres aspectos anteriores, es posible darle una traducción práctica y efectiva al principio de la pertinencia cultural curricular, que es necesario hacer funcionar en relación a todos los componentes de los procesos de aprendizaje. Para esto, debe apoyarse en el hecho, reiteradamente constatado por la sicología etnográfica, de que el grado de familiaridad cultural con los elementos presentes en una situación de aprendizaje ejerce una influencia decisiva sobre las actuaciones de los sujetos. Como lo ha señalado Sternberg (1988) "cuanto más familiar y culturalmente relevante sea una situación, más probable es que las personas ejecuten la tarea de modo adecuado" (1065). Por cierto, esto viene a confirmar la justeza del principio de pertinencia curricular, pero la sicología cultural ha hecho ver que no puede limitarse solamente a los conocimientos del niño, o a algún otro aspecto de la situación de aprendizaje. Esto, debido a que el principio de la familiaridad cultural opera en relación a todos los aspectos de ésta, incluyendo elementos en apariencia insignificantes, como es el caso de los materiales que se emplean en una tarea determinada, los que incrementan de modo considerable las posibilidades de que pueda ser abordada con éxito cuando forman parte del entorno cultural de los niños. Un "efecto de familiaridad con el material" (Cubero y De La Matta, 2005: 66) que funciona también en relación al formato en que un material es presentado, como fotografías, dibujo u objetos reales.

Son numerosas las experiencias implementadas por la sicología cultural que demuestran los efectos de la familiaridad cultural, y que han llevado a concluir que las personas casi siempre "obtienen un rendimiento notable en tareas que son formalmente similares a las tareas en las que no consiguen desempeñarse con éxito en una situación como la de los escenarios tradicionales de los investigadores" (Schliemann et al. 1997: 179). Y es algo que se manifiesta en relación a todo tipo de tareas, como las de conservación de Piaget, las de razonamiento y clasificación, de memorización y cálculo, entre otras; y que se manifiesta, asimismo, en los distintos aspectos de una situación de aprendizaje que han sido examinados antes, incluyendo además la familiaridad con la actividad que requiere una tarea, con lo que a los sujetos "se les pide hacer y cómo deben hacerlo" (Cubero, De La Mata, 2005: 64).

Por ello, desde la perspectiva cultural de la cognición se ha puesto especial hincapié en la necesidad de elaborar escenarios de aprendizaje que sean concordantes con las experiencias culturales de los niños, porque hay "muchos (...) ejemplos de las dificultades que experimentan los niños cuando se les educa en un sistema e intentan funcionar en otro con el que su propio bagaje cultural no les permite tener una familiaridad suficiente" (Rogoff, 1993: 255), lo que constituye sin duda la experiencia más frecuente de los niños indígenas en el ámbito escolar.

El principio esencial de la pedagogía intercultural exige, por lo tanto, adecuar las condiciones y los procesos de aprendizaje, en sus más diversos aspectos y sin excluir ninguno de ellos, a las características culturales específicas de los niños indígenas. Para lo cual es menester considerar, además, la distancia que separa (en cada uno de esos aspectos) las modalidades de existencia y de aprendizaje de los niños, de aquellas que son propias de los espacios escolares. Una distancia que reviste, en este caso, una amplitud extrema, que parece difícilmente reducirse de modo significativo en el marco de las estructuras escolares 
clásicas, pero que sí podría disminuir de manera apreciable inspirándose en el ejemplo de prácticas pedagógicas de los pueblos autóctonos que se aproximan a las de la escuela por la importancia que conceden a la enseñanza.

Al respecto, el ejemplo de los guareños, que habitan en una isla ubicada en el delta del Orinoco, resulta especialmente valioso. Si bien los niños se involucran allí desde pequeños en trabajos agrícolas, en el cuidado de animales domésticos, en actividades de caza y pesca, participan al mismo tiempo en un proceso de instrucción que siempre se estructura en torno a contextos de acción práctica. En cada lección, el instructor se dedica primero a familiarizar a los niños con el entorno en el que se va a realizar la tarea, de la cual proporciona además una demostración. Con posterioridad, se pide a los aprendices resolver secuencialmente una serie de subtareas de un grado de complejidad creciente que forman en conjunto la tarea completa, y en las que cada vez se vuelven a poner en práctica las destrezas previamente adquiridas. Los niños que exhiben un avance más importante prestan ayuda al maestro y pueden seguir practicando por su propia cuenta, hasta que la presencia de éste ya no resulta necesaria (Rogoff, 1993).

Cabe señalar, por último, que la implementación de dispositivos curriculares realmente pertinentes para los niños de los distintos pueblos indígenas requiere necesariamente el apoyo de investigaciones etnográficas, orientadas hacia el estudio de su cultura en todos los aspectos de especial relevancia para la puesta en marcha de los aprendizajes interculturales que se intenta promover, como es el caso de las prácticas sociales de su comunidad, de sus formas de participación en ellas, de sus saberes y destrezas, de sus modalidades de aprendizaje, de las características de la interacción y comunicación con los adultos y los demás niños, de la manera en que es utilizado el lenguaje, de la forma y frecuencia de las narraciones y preguntas, de las modalidades culturales de funcionamiento cognitivo que allí prevalecen.

En todos estos aspectos de la experiencia cultural de los niños, al igual que en otros que no han sido mencionados, cada una de las comunidades indígenas presenta rasgos particulares, de suerte que aquellos que son comunes a los diversos grupos étnicos de América Latina no constituyen en ningún caso una información suficiente para la puesta en marcha de una pedagogía intercultural, la que no puede dejar de considerar el hecho de que cada cultura cuenta también con su propia cultura del aprendizaje.

\section{REFERENCIAS BIBLIOGRÁFICAS}

Amodio, E. (2005). Pautas de crianza entre los pueblos indígenas de Venezuela. Caracas: UNICEF, Fondo de las Naciones Unidas para la Infancia.

Boesch, E.E. (1995). L'action symbolique. Paris: L'Harmattan.

Bossel-Lagos, M. (1989). Savoirs géométriques et arithmétiques de tous les jours chez les tapirapé du Brésil. En Retschizki J., Bossel-Lagos M. y Dasen P.R. (Eds.), La recherche interculturelle (pp. 115-127). Paris: L'Harmattan.

Bril, B. y Lehalle, H. (1988). Le développement psychologique est-il universel?. París: Presses Universitaires de France.

Cole, M. (1999). Psicología cultural. Madrid: Morata.

Chamoux, M.N. (1986). Apprendre autrement. En Rossel P. (Ed.), Demain l'artisanat? (211-335). Paris: Presses Universitaires de France. (1978). La transmission des savoir-faire un objet pour l'ethnologie des techniques? 
Techniques et culture. Bulletin de l'équipe de recherche, vol.191, n.3, 46-83. Paris: Maison des Sciences de l'Hommme.

Cubero, M. y De la Mata, M. (2005). Cultura y procesos cognitivos. En Cubero M. y Ramírez J.D. (Eds.), Vygotski en la psicología contemporánea. Cultura, mente y contexto (pp. 47-79). Buenos Aires: Miño y Dávila.

Greenfield, P. M. y Childs, C.P. (2005). Aprendiendo a tejer en Zinacantán. Un estudio de dos décadas sobre el cambio histórico en la educación informal. Infancia y Aprendizaje, vol. 28, n.1, 3-24.

Lévi-Strauss, C. (1964). El pensamiento salvaje. México: Fondo de Cultura Económica. . (1955). Tristes tropiques. Paris: Plon.

Maccoby, M. y Modiano, N. (1980). Sobre cultura y equivalencia. En Bruner, J.S. (Ed.), Investigaciones sobre el desarrollo cognitivo (pp. 281-293). Madrid: Pablo del Río.

Maybury-Lewis, D. (1992). Millenium: tribal wisdom and the modern world. New York: Viking.

Mishra, R.C. (1997). Cognition and cognitive development. En Berry J.W, Dasen P.R. \& Saraswathi T.S. (Eds.), Handbook of cross-cultural psychology. Volume 2, Basic processes and human development (pp. 143-175). Boston: Allyn and Bacon.

Mohanty, A.K. \& Perregaux, C. (1997). Language acquisition and bilingualism. En Berry J.W, Dasen P.R. \& Saraswathi T.S. (Eds.), Handbook of cross-cultural psychology. Volume 2, Basic processes and human development (pp. 217-253). Boston: Allyn and Bacon.

Paradise, R. (2005). Motivación e iniciativa en el aprendizaje informal. Revista Electrónica Sinéctica, n.26, 12-21. Extraído el 22 de Abril 2014 de http://www.redalcy.org/pdf./998/99815914003.pdf.

Pozo, J. I. (2006). Adquisición del conocimiento. Madrid: Morata.

Quilaqueo, D. (2005). Construcción de un modelo de formación inicial docente en educación cultural bilingüe. En Quilaqueo D., Quintriqueo S. y Cárdenas P. (Eds.), Educación, curriculum e interculturalidad. Elementos sobre la formación de profesores en contexto mapuche (pp. 31-71). Temuco: Facultad de Educación, Universidad Católica de Temuco.

Quintriqueo, S. (2005). Implicancias de la escolarización en la construcción de la identidad cultural de los alumnos mapunche en el medio escolar de la IX Región. En Quilaqueo D., Quintriqueo S. y Cárdenas P. (Eds.), Educación, curriculum e interculturalidad. Elementos sobre la formación de profesores en contexto mapuche (pp. 191-248). Temuco: Facultad de Educación, Universidad Católica de Temuco.

Rogoff, B. (2003). The cultural nature of human development. New York: Oxford University Press. . (1993). Aprendices del pensamiento. El desarrollo cognitivo en el contexto social. Barcelona: Paidós.

Romero, R. y Gottret, G. (2001). Matemática andina: abordaje psicogenético. En A. Lizarzaburu y G. Zapata (Eds.), Pluriculturalidad y aprendizaje de la matemática en América latina. Experiencias y desafios (pp. 124-153). Madrid: Morata.

Schliemann, A.D., Carraher, D.W. \& Ceci, S.J. (1997). Everyday cognition. En Berry J.W, Dasen P.R. \& Saraswathi T.S. (Eds.), Handbook of cross-cultural psychology. Volume 2 Basic processes and human development (pp. 177-216). Boston: Allyn and Bacon.

Sternberg, R.J. (1988). Inteligencia Humana, III. Sociedad, cultura e inteligencia. Barcelona: Paidós. Super, C. \& Harkness, S. (1997). The cultural structuring of child development. En Berry J.W, Dasen P.R. \& Saraswathi T.S. (Eds.), Handbook of cross-cultural psychology. Volume 2, Basic processes and human development (pp. 1-39). Boston: Allyn and Bacon. 
